\title{
POSITIVE BIHARMONIC FUNCTIONS
}

\author{
BRAD BEAVER, LEO SARIO and CECILIA WANG
}

Let $Q$ be the class of quasiharmonic functions $q$, defined by $\Delta q=1, \Delta=d \delta+\delta d$, on a Riemannian manifold. The best source for counterexamples in the $Q$-classification of Riemannian manifolds has been the Poincare $N$-ball $B_{\alpha}^{N}$, that is, the unit $N$-ball $\left\{x=\left(x^{1}, \ldots, x^{N}\right)|| x \mid<1\right\}$ endowed with the Riemannian metric $d s_{\alpha}=$ $\left(1-|x|^{2}\right)^{\alpha}|d x|, \alpha \in \mathbf{R}$. In Sario-Wang [3] it was shown that for each of the classes $Q P, Q B, Q D$, and $Q C$ of $Q$-functions which are positive, bounded, Dirichlet finite, or bounded Dirichlet finite, respectively, values $\alpha$ can be found for which these classes are void. By contrast, the class $Q N$ of negative $Q$-functions on $B_{\alpha}^{N}$ is not void for any $\alpha$. Even the Euclidean plane $\mathbf{R}^{2}$, which is void of any other functions considered in classification theory, trivially carries $Q N$-functions.

It was, therefore, long thought that there may exist no Riemannian manifolds which carry no $Q N$-functions. That this is, however, not the case, was shown in NakaiSario [1].

Trivially, the existence of $Q N$-functions implies that the class $H^{2} P$ of positive nonharmonic biharmonic functions is nonvoid. The natural question thus arose, and has remained unsolved thus far: do there exist Riemannian manifolds which do not carry $H^{2} P$-functions? In the present paper we shall answer this question in the affirmative for any dimension $N \geqq 2$ : the class $O_{H^{2} P}^{N}$ of Riemannian $N$-manifolds which carry no $H^{2} P$-functions is nonvoid. We also show that the inclusion $O_{H^{2} P}^{N} \subset$ $O_{Q N}^{N}$ is strict by constructing a manifold in the delicately "small" class of Riemannian $N$-manifolds which carry $H^{2} P$-functions but nevertheless do not carry $Q N$-functions.

1. Let $S$ be the complex plane with the conformal metric

where $x=r e^{i \theta}$ and

$$
d s^{2}=\lambda(x) d r^{2}+\lambda(x) r^{2} d \theta^{2},
$$

$$
\lambda(x)=1+(3+\varepsilon)(5+\varepsilon)(1-\sin \theta) r^{2+\varepsilon}
$$

(cf. Nakai-Sario [1]). Denote by $\Delta$ the Laplace-Beltrami operator on $S$ and by $\Delta_{e}$ the Euclidean Laplacian. It is clear that $\Delta=\lambda^{-1} \Delta_{e}$. Therefore, harmonic functions on $S$ are the same as harmonic functions on the Euclidean plane, viz. [2],

$$
h(x)=h\left(r e^{i \theta}\right)=\sum_{n=0}^{\infty} r^{n}\left(a_{n} \sin n \theta+b_{n} \cos n \theta\right)
$$


An arbitrary biharmonic function $w$ on $S$ must satisfy $\Delta w=h$. Let $H^{2}$ be the class of nonharmonic biharmonic functions. By a straightforward but somewhat tedious computation we obtain:

Lemma. Every $w(x) \in H^{2}(S)$ can be written as

$$
w(x)=h(x)+c q(x)+\sum_{n=1}^{\infty} c_{n} u_{n}(x)+\sum_{n=1}^{\infty} d_{n} v_{n}(x)
$$

with $\Delta h(x)=0, \Delta q(x)=1, \Delta u_{n}(x)=r^{n} \sin n \theta$, and $\Delta v_{n}(x)=r^{n} \cos n \theta$. Here

$$
\begin{gathered}
h(x)=\sum_{n=0}^{\infty} r^{n}\left(a_{n} \sin n \theta+b_{n} \cos n \theta\right), \\
q(x)=-\frac{1}{4} r^{2}-\left[(3+\varepsilon)(5+\varepsilon)(4+\varepsilon)^{-2}-\sin \theta\right] r^{4+\varepsilon}, \\
u_{n}(x)=-(4 n+4)^{-1} r^{n+2} \sin n \theta-(3+\varepsilon)(5+\varepsilon)\left[(n+4+\varepsilon)^{2}-n^{2}\right]^{-1} r^{n+4+\varepsilon} \sin n \theta \\
-(3+\varepsilon)(5+\varepsilon) r^{n+4+\varepsilon}\left(\alpha_{n} \sin \theta \sin n \theta+\beta_{n} \cos \theta \cos n \theta\right),
\end{gathered}
$$

where $\alpha_{n}, \beta_{n}$ satisfy

and

$$
\left\{\begin{array}{l}
{\left[(n+4+\varepsilon)^{2}-n^{2}-1\right] \alpha_{n}+2 n \beta_{n}=-1} \\
2 n \alpha_{n}+\left[(n+4+\varepsilon)^{2}-n^{2}-1\right] \beta_{n}=0
\end{array}\right.
$$

$$
\begin{gathered}
\eta_{n}(x)=-(4 n+4)^{-1} r^{n+2} \cos n \theta-(3+\varepsilon)(5+\varepsilon)\left[(n+4+\varepsilon)^{2}-n^{2}\right]^{-1} r^{n+4+\varepsilon} \cos n \theta \\
-(3+\varepsilon)(5+\varepsilon) r^{n+4+\varepsilon}\left(\gamma_{n} \sin \theta \cos n \theta+\delta_{n} \cos \theta \sin n \theta\right),
\end{gathered}
$$

where $\gamma_{n}, \delta_{n}$ satisfy

$$
\left\{\begin{array}{l}
{\left[(n+4+\varepsilon)^{2}-n^{2}-1\right] \gamma_{n}-2 n \delta_{n}=-1,} \\
2 n \gamma_{n}-\left[(n+4+\varepsilon)^{2}-n^{2}-1\right] \delta_{n}=0 .
\end{array}\right.
$$

2. We shall show that the manifold $S$ carries no positive nonharmonic biharmonic functions:

Lemma. $S \in O_{H^{2 P}}^{2}$.

Proof. Suppose there exists an $H^{2} P$-function $w(x)$ on $S$. Clearly

$$
\frac{1}{2 \pi} \int_{0}^{2 \pi} w\left(r e^{i \theta}\right)(1 \pm \sin n \theta) d \theta \geqq 0,
$$

where the integral is taken along the circle $|x|=r$. For $n \geqq 2$,

$$
\begin{gathered}
\frac{1}{2 \pi} \int_{0}^{2 \pi} w\left(r e^{i \theta}\right)(1+\sin n \theta) d \theta=b_{0}-\frac{1}{4} c r^{2}-(3+\varepsilon)(5+\varepsilon)(4+\varepsilon)^{-2} r^{4+\varepsilon}+\frac{1}{2} a_{n} r^{n} \\
-\frac{1}{2} c_{n}\left\{(4 n+4)^{-1} r^{n+2}+(3+\varepsilon)(5+\varepsilon)\left[(n+4+\varepsilon)^{2}-n^{2}\right]^{-1} r^{n+4+\varepsilon}\right\} \geqq 0,
\end{gathered}
$$


and

$$
\begin{gathered}
\frac{1}{2 \pi} \int_{0}^{2 \pi} w\left(r e^{i \theta}\right)(1-\sin n \theta) d \theta=b_{0}-\frac{1}{4} c r^{2}-(3+\varepsilon)(5+\varepsilon)(4+\varepsilon)^{-2} r^{4+\varepsilon}-\frac{1}{2} a_{n} r^{n} \\
+\frac{1}{2} c_{n}\left\{(4 n+4)^{-1} r^{n+2}+(3+\varepsilon)(5+\varepsilon)\left[(n+4+\varepsilon)^{2}-n^{2}\right]^{-1} r^{n+4+\varepsilon}\right\} \geqq 0 .
\end{gathered}
$$

On letting $r \rightarrow \infty$ we conclude from these two inequalities that $c_{n}=0$ for $n \geqq 2$, and consequently $a_{n}=0$ for $n \geqq 5$. For $n=1$, we repeat the argument and obtain $c_{1}=0$ and therefore $c_{n}=0$ for all $n$.

Similarly, an integration of $w\left(r e^{i \theta}\right)(1 \pm \cos n \theta)$ gives the corresponding results for the cosine terms: $d_{n}=0$, or all $n$ and $b_{n}=0$, or $n \geqq 5$.

We have reduced the expansion of $w$ to

$$
w(x)=\sum_{n=0}^{4} r^{n}\left(a_{n} \sin n \theta+b_{n} \cos n \theta\right)-c\left\{\frac{1}{4} r^{2}+\left[(3+\varepsilon)(5+\varepsilon)(4+\varepsilon)^{-2}-\sin \theta\right] r^{4+\varepsilon}\right\} .
$$

As $r \rightarrow \infty$,

Since

$$
w(x)=-c\left[(3+\varepsilon)(5+\varepsilon)(4+\varepsilon)^{-2}-\sin \theta\right] r^{4+\varepsilon}+O\left(r^{4}\right) .
$$

$$
\begin{aligned}
& (3+\varepsilon)(5+\varepsilon)(4+\varepsilon)^{-2}-\sin \pi / 2<0, \\
& (3+\varepsilon)(5+\varepsilon)(4+\varepsilon)^{-2}-\sin (-\pi / 2)>0
\end{aligned}
$$

and $w\left(r e^{i \theta}\right) \geqq 0$ for all $r, \theta$, we must have $c=0$. Thus

$$
w(x)=\sum_{n=0}^{4} r^{n}\left(a_{n} \sin n \theta+b_{n} \cos n \theta\right)
$$

a harmonic function. Furthermore, $w$ reduces to a constant since a parabolic manifold $S$ does not carry nonconstant positive harmonic functions.

3. From the above case of dimension 2 we proceed to the construction of an $N$-dimensional, $N \geqq 2$, manifold which carries no positive nonharmonic biharmonic functions (cf. Nakai-Sario [1]).

Theorem. $O_{H^{2} P}^{N} \neq \emptyset, N \geqq 2$.

Proof. We may assume $N>2$. Let $S$ be as in Section 1 , and take any compact bordered $(N-2)$-manifold $T$, with local coordinates $y=\left(y^{1}, \ldots, y^{N-2}\right)$, say, and with $\partial T=\emptyset$. For our purposes it suffices to consider the special case of the torus $\left|y^{i}\right|<1, i=1, \ldots, N-2$, with each $\left\{y^{i}=1\right\}$ identified with $\left\{y^{i}=-1\right\}$. Its border $\partial T=\emptyset$ can be reviewed as an oriented compact $(N-3)$-manifold traced in opposite 
directions. Endow the product manifold $S \times T$ with the metric

$$
d s^{2}=\lambda(x) d x^{2}+d y^{2} .
$$

The Laplace-Beltrami operator $\Delta$ on $S \times T$ is then

$$
\Delta=\Delta_{x}+\Delta_{y}
$$

where $\Delta_{x}$ and $\Delta_{y}$ are the Laplace-Beltrami operators on $S$ and $T$, respectively. We shall show that $S \times T$ carries no $H^{2} P$-functions whenever $S$ carries none. Suppose $S \times T \notin O_{H^{2} P}^{N}$ and let $w(x, y) \in H^{2} P(S \times T)$. Define

$$
w_{0}(x)=\int_{T} w(x, y) d y
$$

on $S$. In view of $\partial T=\emptyset$, we have

$$
\begin{gathered}
\Delta_{x} w_{0}(x)=\int_{T} \Delta_{x} w(x, y) d y=\int_{T}\left(\Delta-\Delta_{y}\right) w(x, y) d y=\int_{T} \Delta w(x, y) d y-\int_{T} \Delta_{y} w(x, y) d y \\
=\int_{T} \Delta w(x, y) d y+\int_{\partial T} *_{y} d_{y} w(x, y)=\int_{T} \Delta w(x, y) d y .
\end{gathered}
$$

By $\Delta^{2} w=0$,

$$
\begin{gathered}
\Delta_{x}^{2} w_{0}(x)=\int_{T} \Delta_{x}(\Delta w(x, y)) d y=\int\left(\Delta-\Delta_{y}\right)(\Delta w(x, y)) d y \\
=-\int_{T} \Delta_{y}(\Delta w(x, y)) d y=\int_{\partial T} *_{y} d_{y}(\Delta w(x, y))=0 .
\end{gathered}
$$

Thus $w_{0}(x) \in H^{2}(S)$. Obviously, $w_{0}(x)$ is positive whenever $w(x, y)$ is. Therefore, $S \times T \notin O_{H^{2 P}}^{N}$ implies $S \notin O_{H^{2} P}^{2}$, a contradiction.

4. Next we assert:

Theorem. The strict inclusion

holds for all $N \geqq 2$.

$$
O_{H^{2} P}^{N}<O_{Q N}^{N}
$$

Proof. Remove from the complex plane the closed unit disk and consider the remaining manifold

$$
S_{1}=\{(r, \theta) \mid 1<r<\infty,-\pi \leqq \theta \leqq \pi\}
$$

endowed with the conformal metric $d s^{2}=\lambda(x) d x^{2}$ as in Section 1. We shall first show that the manifold $S_{1}$ carries $H^{2} P$-functions but no $Q N$-functions.

Every $w \in H^{2}\left(S_{1}\right)$ has an expansion

$$
w(x)=h(x)+c q(x)+d p(x)+\sum_{n \neq 0}\left[c_{n} u_{n}(x)+d_{n} v_{n}(x)\right],
$$


where $q(x), u_{n}(x), v_{n}(x)$ are as in Section 1 for $n \neq-1$, and

$$
\begin{gathered}
h(x)=a \log r+\sum_{n=-\infty}^{\infty} r^{n}\left(a_{n} \sin n \theta+b_{n} \cos n \theta\right), \\
p(x)=-\frac{1}{4} r^{2}(\log r-1)+(3+\varepsilon)(5+\varepsilon)(4+\varepsilon)^{-2}\left[2(4+\varepsilon)^{-1}-\log r\right] r^{4+\varepsilon} \\
+\left\{\log r-2(4+\varepsilon)\left[(4+\varepsilon)^{2}-1\right]^{-1}\right\} \sin \theta r^{4+\varepsilon}, \\
u_{-1}(x)=-\frac{1}{2} r \log r \sin \theta+(3+\varepsilon)(5+\varepsilon)\left[(3+\varepsilon)^{2}-1\right]^{-1} r^{3+\varepsilon} \sin \theta \\
-(3+\varepsilon)(5+\varepsilon) r^{3+\varepsilon}\left(\alpha_{-1} \sin ^{2} \theta+\beta_{-1} \cos ^{2} \theta\right), \\
v_{-1}(x)=-\frac{1}{2} r \log r \cos \theta-(3+\alpha)(5+\alpha)\left[(3+\varepsilon)^{2}-1\right]^{-1} r^{3+\varepsilon} \cos \theta \\
-(3+\varepsilon)(5+\varepsilon) r^{3+\varepsilon}\left(\gamma_{-1}-\delta_{-1}\right) \sin \theta \cos \theta,
\end{gathered}
$$

where $\alpha_{-1}, \beta_{-1}, \gamma_{-1}$, and $\delta_{-1}$ are as in Section 1 for $n=-1$. In view of Section 1 , $\Delta u_{n}(x)=r^{n} \sin n \theta$ and $\Delta v_{n}(x)=r^{n} \cos n \theta$. Clearly $u_{n}(x)$ and $v_{n}(x)$ are biharmonic. By virtue of $u_{n}(x)=O\left(r^{n+4+\varepsilon}\right)$ and $v_{n}(x)=O\left(r^{n+4+\varepsilon}\right), u_{n}(x)$ and $v_{n}(x)$ are bounded if $n \leqq-5$. By adding an appropriate constant $c$, we obtain a function $w_{0}(x)=$ $u_{n}(x)+c$ or $v_{n}(x)+c$ which belongs to $H^{2} P\left(S_{1}\right)$, so that $S_{1} \in \tilde{O}_{H^{2} P}^{2}$; here and later $\widetilde{O}$ stands for the complement of $O$.

In order to show that $S_{1} \times T \in \widetilde{O}_{H^{2} P}^{N}$, define

Since

$$
w_{1}(x, y)=w_{0}(x), \quad(x, y) \in S_{1} \times T .
$$

$$
\Delta^{2} w_{1}(x, y)=\left(\Delta_{x}+\Delta_{y}\right)^{2} w_{0}(x)=\Delta_{x}^{2} w_{0}(x)=0,
$$

and $w_{1}$ is obviously positive, $w_{1} \in H^{2} P\left(S_{1} \times T\right)$.

To show that $S_{1} \in O_{Q_{N}}^{2}$, we reason as in Section 2. The conclusion $S_{1} \times T \in O_{Q_{N}}^{N}$ then follows as in Section 3. Thus we have $S_{1} \times T \in O_{Q_{N}}^{N} \cap \widetilde{O}_{H^{2} P}^{N}$, hence the Theorem.

5. We have seen that the manifold $S=\{|x|<\infty\}$, with the Riemannian metric $d s^{2}=\lambda(x) d x^{2}, \lambda(x)=1+(3+\varepsilon)(5+\varepsilon)(1-\sin \theta) r^{2+\varepsilon}$, carries no $H^{2} P$-functions. On the other hand, the manifold $S_{1}$ obtained by deleting the unit disk from $S$ does carry $H^{2} P$-functions. It is perhaps of some interest to investigate the intermediate manifold $S_{0}=\{0<|x|<\infty\}$ with the same Riemannian metric as $S$ and $S_{1}$. Even though every $w \in H^{2}\left(S_{0}\right)$ has the same expansion as $w \in H^{2}\left(S_{1}\right)$, repeating the reasoning in Section 2 leads to the conclusion that, contrary to the case of $S_{1}$, the manifold $S_{0}$ carries no $H^{2} P$-functions. Thus $S_{0}$ is another counterexample to show the nonvoidness of $O_{H^{2} P}^{2}$, and hence also of $O_{Q N}^{2}$. An analogue of the argument in Section 3 extends the conclusion to an arbitrary dimension. 


\section{References}

[1] NAKaI M., and L. SARIo: Existence of negative quasiharmonic functions. - Jubilee volume dedicated to the 70th anniversary of Academician I. N. Vekua, Academy of Sciences of the USSR, Nauka, 1978, 413-417.

[2] SARIo, L., M. NAKaI, C. WANG and L. ChUNG: Classification theory of Riemannian manifolds. - Lecture Notes in Mathematics 605, Springer-Verlag, Berlin-Heidelberg-New York, 1977.

[3] SARIO, L., and C. WANG: Quasiharmonic functions on the Poincaré N-ball. - Rend. Mat. (6), 1973, 1-14.

University of California, Los Angeles

Department of Mathematics

Los Angeles, California 90024

USA

Received 10 July 1981
Arizona State University

Department of Mathematics

Tempe, Arizona 85281

USA 